\title{
Protée
}

\section{Un hjelmslévien en visite chez un peircéen}

\section{Jean Fisette et Fernand Roy}

Volume 27, numéro 2, 1999

La réception

URI : https://id.erudit.org/iderudit/030564ar

DOI : https://doi.org/10.7202/030564ar

Aller au sommaire du numéro

Éditeur(s)

Département des arts et lettres - Université du Québec à Chicoutimi

ISSN

0300-3523 (imprimé)

1708-2307 (numérique)

Découvrir la revue

Citer cet article

Fisette, J. \& Roy, F. (1999). Un hjelmslévien en visite chez un peircéen. Protée, 27(2), 118-124. https://doi.org/10.7202/030564ar d'utilisation que vous pouvez consulter en ligne.

https://apropos.erudit.org/fr/usagers/politique-dutilisation/ 


\title{
UN HJELM SLÉVIEN EN VISITE CHEZ UN PEIRCÉEN
}

\author{
Échange à partir de Pour une pragmatique de la signification *
}

FERN AN D ROY, U niversité du Q uébec à C hicoutimi

JEAN FISETTE, U niversité du Q uébec à M ontréal

\begin{abstract}
Contexte (Fernand Roy). A prèsavoir lu crayon en main et avec beaucoup d'intérêt Pour une pragmatique de la signification, j'ai eu le goût de discuter avec J ean Fisette: d'une part, il me semblait à l'occasion injustement sévère à l'égard de la perspective hjelmstévienneet, d'autre part, jeme reconnaissais souvent dans les mi ses à l'essai sur les textes littéraires qu'il faisait des catégories peircéennes. En décembre 1996, je lui ai donc proposé que nousinteragissions au cours de l'hiver par courrier électronique: jelui soumettrais d'entrée de jeu une série de questions pour lui donner à voir cequi me préoccupait; mais sour éviter quel'échange soit trop linéaire, il était convenu queje reformulerais une à une mes questions en cours de route, me permettant ainsi de réagir à sa (ses) réponse(s) antérieure(s). Au début de juin 1997, nous nous sommes retrouvés avec une correspondance de 60 pages. Pour en arriver à un texte recevable par la revueP rotée - l'idée était là au départ!-, après un été de réflexion, j'ai reformulél'ensemble de mon propos en cinq questions que j'ai transmises à J ean Fi sette et auxquelles il a réagi en une seule fois. Les titres précédant les questions n'ont pas été pensées en vue d'une publication, ils font partie de la formulation initiale.
\end{abstract}

\section{Un peu d'épistémologie}

FR Au début de votre livre, vous parlez d'une «absolue opposition » entre la théorie sémiotique - pragmatique- de Peirce et celle- la glossématique- deH jelmslev. Jenepense pas nécessairement commevous- jenecrois pasàl'absolu!maisje suis bien forcéd'admettrequ'il y a dans votrepropos plus qu'une simple insistance didactique: au-delà des tentatives d'Eco et de D eledalle, vous rejetez la possibilité d'uneéventuellerencontre entrela trichotomique (priméité, secondéité et tercéité) de l'un et la «tétratomique» (substance de l'expression, forme de l'expression, substance du contenu et forme du contenu) de l'autre. Ce qui revient à dire - et je suis plutôt d'accord, théoriquement- que, en toute logique, il faut reconnaître la pertinence - en matière de construction d'objet de savoir - du principe de contradiction. J'arrive cependant à moins bien vous suivre quand, pour étayer votre référence au faillibilisme de Peirce, vous me paraissez déformer le propos de H jelmslev - ce que vous appelez son positivisme- en lui reprochant d'avoir prétendu qu'unethéorienepeut jamaisêtre démontrée et que, partant, elle vaut jusqu'à preuve du contraire, c'est-à-dire jusqu'à ce qu'elle soit contredite par les faits - principe de contradiction - ou si ellen'arrive pas à rendre compte d'une partie des faits dont elle devrait normalement rendre compte principed'exhaustivité. M a premièrequestion est la suivante: comment pouvez-vous prétendre que la théorie de Peirce est plus opérationnelle, si vous nefondez pas votrejugement sur leprincipe decontradiction et sur celui del'exhaustivité? $\mathrm{N}$ e serait-il pas plus conforme à votre pensée de prétendre simplement que vous trouvez que la théorie de Peirce est plus opérationnelle que celle de $\mathrm{H}$ jelmslev?

JF II est en effet assez évident que dans les premiers textes quej'ai écrits sur la sémiotique de Peirce (dont les chapitres 1 et 4 de la Pragmatique), j'aie pu me prêter à une certaine rhétorique pour souligner à doubletrait, pourrait-on dire, la 
différence fondamentale entre les deux projets sémiotiques auxquels vous vous référez. M ais au-delà de ces effets de discours, il est impérieux de reconnaître les divergences fondamentales entre la sémiotique de Peirce et les sémiologies structuralistes issues des travaux de Saussure et de $\mathrm{H}$ jelmslev. J'irai plus loin: jesuis convaincu quec'est le «gommage» de ces divergences fondamentales qui est responsable deladilution dela pensée de Peirce, desa réduction àquel ques notions décontextualisées et devenues simplement utiles, comme la série «icône - indice - symbole» ou encore le schéma binaire «token-type», puis à la non-prise en compte réelle de ce qui fait l'apport original de la «semeiotic».

$\mathrm{Si}$ je tentais de ramener à quelques traits fondamentaux ces divergences, je proposerais ceci : Peirce, se définissant comme logicien, s'inscrit sur le terrain de la recherche cognitive (il emploie d'ailleurs ce mot) al ors que Saussure, se présentant comme linguiste, se donne un projet strictement descriptif et explicatif; Saussure et $\mathrm{H}$ jelmslev cherchent à donner une représentation - et donc une explication- des systèmes de signification suivant le modèle de la langue, sur une base strictement synchronique (je n'ai pas le temps de reprendre les métaphores toujours spatiales qui sont extrêmement révélatrices), alorsque chez Peirce, la notion de sémiose définit le signe comme une inférence, un processus de transformation qui s'inscrit nécessairement sur un axe temporel. Enfin, l'aspect de cette question qui me paraît le plus important tient à la place du sujet observateur: les discours de Saussure et de $\mathrm{H}$ jelmslev présupposent un sujet observateur neutre, extérieur à l'objet analysé, non focalisé (au sens que donna jadis $G$ enette à ce terme); et I'on sait que c'est précisément cettedéfinition épistémologiqued'un «sujet détaché» qui justifie les critères d'exhaustivité et de noncontradiction permettant de val ider l'analyse; dans la logique dePeirce (qui avait pourtant uneformation de scientifique), le sujet-analyste, étant placé à l'intérieur de son objet d'analyse, n'en a jamais de vue globale et synthétique, d'où la reconnaissance du «faillibilisme» et l'absence du recours au critère d'exhaustivité pour valider l'analyse. À ce propos, le fragment intitulé «La conscience ressemble à un lac sans fond » est particulièrement significatif.

$D$ ans la mesure où je suis convaincu que tout processus de production designification - et la lecture en est un castypeprésuppose, de la part du sujet, une position d'immersion de la conscience à l'intérieur de la masse des signes portés par l'objet à l'étude - justement parce que ce sont des signes et non des signaux ou de simples stimuli - , mon choix pour la problématique de Peirce dépassenécessairement le simple caractère «opérationnel » auquel vous vous référez. $C^{\prime}$ 'est pour moi un fait de nécessité logique.

\section{La notion de trichotomique et celle de code}

FR Si je dis - reprise de la formulation de Todorov et D ucrot- qu'un signifié est ce qui manque à un signe pour qu'il y ait signification, est-cequejene respecte pas la fameuse exigence de Peirce au sujet de la relation triadique? Est-ce quelefait d'entendre «code» comme résultat d'un consensus antérieur sur lequel table tout processus sémiotique n'entrâne pas la nécessité de penser quela re-mise en circulation d'un signen'est possibleque parce que la lecture constitutive d'un signe est par définition produite par un autre et que c'est cettelecture d'un autrequi rend leprocessus sémiotique interminable?

JF Pour répondre brièvement à cette question, je me référerai à la distinction que j'avais déjà proposée dans mon Introduction* entre les termes «sens» et «signification». Le «sens» d'un signe découle de son appartenance à un code (Levi-Strauss écrivait, quel que part, quele sens d'un termese ramène à la place qu'il occupe dans une structure). Le mot «signification», en raison de son suffixe «-ion» désigne une action, soit l' «action de signifier»; cette idée de l'action est nécessairement liéeàla «circulation du signe», à son incessante «re-miseen circulation», commevous leproposez avec justesse et que, dans le vocabulaire de Peirce, on nomme «un mouvement jamais achevé de sémiose». La lecture est effectivement un processus de «signification», illustrant ce caractère fondamentalement infini de la sémiose. Je pense que les acquis enregistrés par un signe, au cours de son «vagabondage dans les trois univers» (D eledalle) que représente une lecture donnée, débordent, par définition, les acquis antérieursqui sont déjà là, inscrits dansle «code» dela langue et que l'on désigne sous le terme saussurien de «signifié» ou encoresous la formule hjelmslévienne de «formedu contenu». Je suis globalement d'accord avec votre analyse où vous inscrivez le rôle de l'autre et de l'échange dans le processus de constitution du signe; je reviens cependant sur un petit détail: si on définit le signe comme une inférence, un mouvement, alors le terme «signifié», pris dans son acception saussurienne, marquel'arrivée à un terme et donc l'arrêt du mouvement. C'est pourquoi lemot «signifié» est total ement contradictoire avec le terme «interprétant» et, en toute rigueur, l'on ne saurait lui trouver d'équivalent dans la «semeiotic».

* Jean Fisette, Introduction à la sémi otique de Charles S. Peirce, M ontréal, XYZ, 1990. 


\section{La socialité de la signification}

FR À quelques occasions (pas assez nombreuses, à mon goût) vous parlez de la «socialité de la signification». Certaines de vos formules me semblent ouvrir des perspectives très intéressantes («terreau consensuel»; «la sémiose comme partage d'un signe inachevé»; "cette position pragmatiste, définie sur une base purement logique, ramène la signification au consensus social », p. 149). M aispourquoi entendez-vous comme «base purement logique» une position qui définit la signification commerésultante, sur un matériau donné, d'un processus interactif qui tire son sens du fait de produire un consensus à partir de positions au départ contradictoires (celles d'un «je» et d'un «tu»)? Cemesemble à tout le moins une base éminemment dialectique, en ce qu'elleimpliqueque la signification, loin d'arrêter la sémiose, la boucle plutôt, assurant dès lors la possibilité d'une relance à partir d'un consensus - d'où l'idée de hiérarchie, si je saisis bien, même si je ne suis pas d'accord pour hiérarchiser les faits de langage. T oujours les usages ultérieurs - à cause du contexte et des usagers- vont faireévoluer les significations; aussi longtempsqu'il y aura un «tu » récepteur différent d'un «je»locuteur. Est-cequePeirce considèrequela signification apparaît quand le locuteur finit par sanctionner l'interprétation de sa «parole» qui a été anticipée par l'interlocuteur? Y a -t-il dans sa théorie autre chose que la notion d'habitude pour en parler, de la signification entendue au sens de boucle? Et en quoi cette habitude sociale codée estelle à distinguer de la notion d'interprétant? (Je ne vois toujours pas bien comment Peirce arrive à faire l'économie d'un quatrième temps, sauf à parler d'interprétant final ou pragmatique, et à le faire en termes de boucle aussi.)

JF V ousécrivez votre refus de hiérarchiser les faits de langage; je partage entièrement votre position et ma critique vis-à-vis de la notion de «méta-langage» en constitue une démonstration assez probante. Par contre, le terme «hiérarchie» auquel je me réfère fréquemment désigne toujours le modèle des relations de présupposition entre les trois caté gories phanéroscopiques: j'ai proposé, au début du chapitre 7 , une présentation que j'ai voulu très didactique de ce modèle logique. Ce qui est donc hiérarchisé, ce ne sont pas des faits de langage, mais bien des niveaux strictement logiques. Par contre, cette hiérarchie se définit sur une base purement arithmétique et ellen'implique aucune échelle de valeur. Elle désigne, plus simplement, des positions par rapport aux processus d'abstraction (allant vers la tercété) et de concrétion (allant vers la primété). Ainsi, l'une des propositions majeures de mon ouvrage concernant I'objet artistique, c'est que le caractère d'iconicité (donc de niveau premier) y est prédominant; bien plus, la métaphore saisie non pas comme un simple trope, mais comme un processus inférentiel, est incompréhensible en dehors d'une analyse qui prend en compte cette phase d'abolition des différences; en cesens, elles'inscrit comme une plongée dansle «vague», bref dans la priméité; j'ajouterais que je suis assuré qu'à ce point de l'analyse, nous ne sommes pas très éloignés de la "substance du contenu» suivant l'acception que donne $\mathrm{H}$ jelmslev à ce terme. C'est donc dire que le modèle de la hiérarchie des trois grandes catégories serait plutôt à penser en termes d'une topique (comme celles que l'on trouvechez Freud, par exemple), mais le mot «topique», du point de vue étymologique, renvoie aussi à une métaphore spatiale; alors je parlerais plutôt de moments, de phases au cours du mouvement de la sémiose qui fait la signification. Le chapitre 6 porte, en majeure partie, sur de tels mouvements de sémiose en regard de divers textes.

En ce qui concerne le caractère social de la signification, il me semble que ce thème soit omniprésent tout au long de mon étude. Jesuis mêmealléjusqu'à proposer que «socialité» et «signification» soient, pour le sémioticien, des termes synonymes. Je voudrais cependant simplement ici attirer votreattention sur un passage crucial de mon étude (p. 2256 ) - et dont je crains qu'il passe inaperçu, relégué dans un simple paragraphe- où je propose que l'imaginaire, loin d'appartenir strictement aux fantasmes d'un individu isolé, est un territoire commun, partagé par la collectivité et que cette appartenance commune est nécessaire pour expliquer que les rêves, les contes, les poèmes soient compris par les membres de la communauté.

\section{La spatialité constitutive des signes}

FR J'ai beaucoup apprécié le $7^{\text {e }}$ chapitre de votre livre («Représentation, iconicité et pragmatisme»), et tout particulièrement les pages 160 à 175 qui me semblent centrées vers la belle formule «sensation raisonnable» que vous liez à la résolution du paradoxe du «signe [qui], dans sa fuite en avant risque constamment de nous échapper » al ors que «la signification n'est possible que sur la base d'une représentation où l'iconicité est toujours présente [...] ce qui implique une certaine stabilité du representamen » (p. 169). Q uand vous revenez rétroactivement sur votre point de départ (où vous affirmiez qu'une théorie du texte implique quelques 
conditions épistémologiques, dont l'idéeque le signe est par définition mouvement, processus, et qu'en tant que tel il est défini dans un continuum temporel plutôt que dans un découpage spatial, p. 29), n'avez-vous pas l'impression que leproblème dit dela représentation oblige à reconnaître que lesigne-au sens de representamen - est un compositespatiotemporel re-mis en mouvement?

Je vous pose la question pour deux raisons: d'une part, l'idée de signe étendu a besoin de demeurer «relative» - par opposition à «absolue»- et, d'autre part, je ne vois pas comment on peut faire l'économie de la notion d'espace pour intégrer dans le temps ce qui est devenu habitude, soit le résultat de l'apport du «tu», de sa lecture de la «parole» du «je».

JF V ous vous référez à ce passage du premier chapitre où j'ai introduit une distinction forte entre «découpage spatial » et «continuum temporel ». Ce texte est le premier quej'aie écrit sur Peirce, antérieurement à la rédaction de mon Introduction. J'y prenais cette position forte pour m'aider à mieux saisir et marquer de façon plus nette la spécificité de la dé marchepeircéenne et accentuer quel que peu le contrasteavec les sémiologies structurales.

Relisez attentivement ces quelques pages (p. 24ssq.), attachezvous à la métaphore des îlots et de l'archipel où je suggère que «cequi fait sens», cenesont pas lesîlots pris séparément, puis additionnés, ni une saisie statique de l'archipel représenté dans sa totalité, comme sur une carte (ce serait alors une simple structure vue de l'extérieur par un «sujet détaché», comme l'exprime clairement l'expression: «vue à vol d'oiseau»); j'écrivais que ce qui fait sens, c'est bien «[...] la conscience du mouvement, nécessairement temporel, de la vision sautant d'un îlot à un autre, ce moment même où naît la conscience d'un enchaînement ou d'une inférence». Puis, pour me donner une compréhension encore plus forte de ce phénomène, j'ajoutai une autre métaphore, musicale celle-là, parce que le facteur temps y est déterminant: «[...] pensonsà notre perception lorsque nous écoutonsunefugue deJ.S. Bach; ce ne sont pas les notes séparées qui font sens, ni la totalité de la pièce saisie comme structure, mais cette conscience, immanente à l'écoute, de l'enchaînement des notes, des voix et des autres procédés musicaux, en somme del'inférencequi relieles sonsentreeux»; aujourd'hui, j'ajouterais à cette phrase: et qui seule fait un continuum sonore, c'est-à-direla musique.

II devient alors clair que la distinction forte que je tentais d'inscrire concerne moins le schéma temps/ espace que le schéma continuité/discontinuité. J'opposais le continuisme, fondamental chez Peirce, au principe dela discontinuitéque jadis Saussure plaça au centre de sa linguistique sous leterme de différence et qui constitua, comme on le sait, I'une des notions essentielles au fondement dela méthodestructurale. Les métaphores courantes m'ont entraînéà rattacher la continuité au temps et la discontinuitéà l'espace. $0 \mathrm{r}$ on sait bien qu'un substrat sonore, commela musique, donc fondamentalement temporel, est, à un certain niveau, discontinu, comme à un certain niveau de l'analyse de la matière, il n'y a que des discontinuités; mais lequestionnement sémiotique ne se situe pas à ce niveau et on ne peut se permettre de commettre cette erreur. Avez-vous déjà joué un instrument de musique et donc lu une partition? Interpréter une partition, c'est traduire des notes, c'est-à-dire des marques visuelles et graphiques, discontinues, en un continuum sonore. Autre exemple: les systèmes modernes de reproduction sonore, dits numériques, travaillent par définition au niveau du discontinu. Et, à l'inverse, on sait qu'un espace peut être imaginé comme pure continuité sans rupture. Et pourtant, il faut reconnaître, avec des travaux scientifiques probants à cet effet, qu'il y a des prédominances: I'œil est beaucoup plus discriminatoire alors que l'ouïe est beaucoup plus synthétique! D 'où, fort probablement, I'origine de ces associations (continuité- temporalité et discontinuité - spatialité) fondant les métaphores sur lesquelles je me suis appuyé.

Je reviens à la question plus précise que vous posez: effectivement «je» et «tu», les deux interlocuteurs occupent nécessairement des positions spatialement séparées. $M$ aisc'est de la rencontre de leur esprit et du mouvement de pensée qui s'y effectuequesurgit lesigne. Sur cettequestion précise, Peirce a fait une suggestion extrêmement intéressante que vous trouverez dans un court fragment quej'ai cité (chapitre 9 , note 20, p. 223): on y lit que les deux interlocuteurs d'un échange sont «...nécessai rement distincts», alors que, «... dans le signe, ils sont, pour ainsi dire, soudés» («In the Sign they are, so to say, welded», C.P. 4.551). C ette soudure des deux sujets rejoignant ce que Peirce appelle le $\mathrm{M}$ ind constitue en quelque sorteun lieu-tempsoù s'élaborent et setransforment les signes; à partir du moment où ce lieu-temps a été établi, les signes qui l'habitent n'ont plus d'existence que comme inférence, comme mouvement, comme processus en continuelle transformation, d'où la nécessité de poser en prédominancel'axe temporel pour bien désigner le contexte de ce caractère fluide des signes. 
J'ai retenu, d'un fragment trèstardif de Peirce, l'idée du signe étendu qui dénomme en fait le processus de la sémiose alors quele terme «complexe» qualifie non pas le signe mais bien son objet. L'idée de «signe étendu» repose, comme vous l'écrivez fort justement, sur le principe de la relativité des représentations; j'ajouterais leur nécessaire instabilité et, comme je viens de l'écrire, leur fluidité. M ais le principe de la relativité, pris en lui-même, ne peut pas, à son tour, être relativisécar alors tout s'effondrerait dansun relativismetotal. II y a là une position épistémologique de fond, incontournable, qui est au fondement même de la démarche sémiotique.

\section{En marge de la finale d'un poème et de la notion de tercéité}

FR J'ai beaucoup apprécié votre lecture de la finale d'un poème de Saint-D enys $G$ arneau; mais je suis un peu de meuré sur ma faim. Pour deux raisons un peu différentes. Q uand vous parlez de dépassement du seuil linguistique - à mon avis, tout usage constitue un dépassement de ce seuil, si infime soit-il: on ne parle pas le dictionnaire, comme vous le dites si bien!, ce qui marque bien que l'écriture modifie tout de même les usages... - quand vous parlez de dé passement du seuil linguistique, dis-je, vous montrez qu'à un moment donné prend forme (vous vous écrivez «alieu», cequi donneà penser quela notion deformen'a d'existence que dans I'espace, l'autre aidant!) une médiation - tercéitéqui tient à ce que «l'interprétant («enveloppement de la séduction» en l'occurrence) vient générer un objet dynamique qui tient dans la continuité nature/ culture» (p. 231). Si je vous ai bien lu, cette interprétance en acte à la fois fait signe et appelle une nouvelle lecture- la rend nécessaire, sauf à ne pas prendreen compte ce qui est écrit. Est-ce qu'à votre avis cet appel d'une nouvelle lecture s'entend par rapport à un point de départ? par rapport à une habitudequele poème visait à faire évoluer? En d'autres mots: même si votre but n'était pas de rendre compte de l'ensemble du poème, puisque, selon Peirce, tercété présuppose et secondéité et priméité, est-ce que, selon vous, on pourrait identifier dans le poème unerelation triadiqueimpliquant le début, lemilieu et la fin du poème (fin à laquelle vous vous en êtes tenu étant donné votre intention)? C'est tout de même cela le principe de la trichotomique, non? Est-ce que le respect de ce principe n'implique pas, en l'occurrence du poème, à reconnaître qu'unecomposante spatialeest aussi à considérer? J'en viens à ma seconde raison. À mon sens, vous avez bien raison de préciser qu'il ne faut pas voir dans «l'enveloppement de la séduction » le simple terme positif de l'inversion des valeurs du manque posées au départ. J e ne pense cependant pas que Levi-Strauss ait jamais voulu une telle mise à plat; mais enfin, cela se pratique dans certains coins, quand on fait commesi on pouvait réduire la sémiotique à une sémantiqueimmatérielle, c'est-à-dire sans composanteénonciative. D 'un point de vue hjelmslévien - mais non orthodoxe-, ce que vous identifiez comme tercéité dans le poème correspond tout de même à une sorte de retour à un point de départ transformé... en ce sens que ce point d'arrivée du poème déborde nécessairement - ces mots sont de vous- les mots présents dans le poème! Pour rendre compte de ce dé bordement, il me paraît personnellement utile de faire intervenir la notion deformedu contenu (designifié), notion qui n'a évidemment de sens qu'en regard d'une forme de l'expression (d'un signifiant), mais qui ne doit pas être «fondue» avec elle, analytiquement parlant. Je vous donne un exemplesimplistepour bien vousfairevoir ce quejeveux dire, avant de poser ma (deuxième partie de) question. Soit une fiction d'interaction verbale minimale qui serait à l'origine du sens généralement attribué dans notre culture à «toc toc»: des coups réguliers frappés à une porte auxquelsa répondu l'ouverture de la porte par l'interlocuteur. Si on suppose qu'en eux-mêmes les coups ne veulent rien dire et qu'ils deviennent «signifiants» pour un interlocuteur, quand un interlocuteur y anticipe une demande d'ouverture, on peut remarquer que l'ouverture de la porte constitue du POINT DE VUE de l'interlocuteur la substance du contenu ou l'interprétation qu'il actualise très matériellement du signifiant. À cetteinterprétation en acte, va réagir celui qui a frappé - le locuteur - et sa réaction va, s'il entre, constituer une sanction de la lecture faite par l'interlocuteur. $0 \mathrm{n}$ peut dire que l'entrée de celui qui a frappé constitue un retour au point de départ, mais transformé. II me paraît cependant plus opératoire de remarquer que cette entrée constitue le signifié qui manquait au signe - les coups réguliers sur la porte- pour qu'il y ait signification. Si je regardele processus dans son ensemble, il est à mon avis évident que la signification est un résultat qui transcende le processus même et que l'on va retrouver ensuite sous forme de «code» ou d'habitude ou de socialité. Et il me semble que si l'on essaie de lire l'entrée du locuteur au niveau «linguistique», on manque la sémiose, qui en l'occurrence tient à la mise en continuité, l'interlocuteur aidant, des coups comme signifiant et de l'entrée comme signifié (ou forme du contenu). 
(Comme vous pouvez le remarquer par ailleurs, à mon sens la division du plan du contenu en substance et en formen'a de sens que dans une perspective interactive: I'interlocuteur fournit la substance et c'est par l'intermédiaire de l'autre que I'on accède à la «forme»). M a question, maintenant. II me paraît évident que le sémiotique se situe au niveau de la «demande» (la culture) et non au niveau del'entrée (la nature). Pour moi, l'entrée en elle-même n'est pas sémiotique; elle l'est seulement parce que mise en rapport à l'ouverture qui, elle-même, est seconde par rapport aux coups frappés. La seule façon quej'ai - $\mathrm{H}$ jelmslev aidant- d'entendre l'entrée comme forme du contenu, c'est de ne pas oublier que son correspondant est dans la reconnaissance d'une forme de l'expression danslescoups, dansla substance des coups. C ela, ce second moment qui revient à l'interlocuteur, me paraît impliquer cet interlocuteur doublement: c'est en tant qu'interlocuteur réagissant (1) qu'il va ouvrir (2). Et son geste est une réponse autant pour lui-même que pour celui qui a frappé. Est-ce cela - autant pour lui-même que pour celui qui a frappé- pour vous la secondéité? Si c'est le cas, alors, n'ai-je pas un peu raison de dire que, de mon point de vue, votre lecture de la finale correspond dans mon langage à moi à la forme du contenu ou au signifié en tant que ce qui manquait au signifiant pour qu'il puissey avoir signification sous forme de «serait», c'est-à-dire à la condition que quelqu'un d'autre en effectue la lecture?

JF Sur cette idée du dépassement du seuil linguistique, je me contenterai d'ajouter à ce que vous en dites (et que je partage) que, par là, je cherche à me dégager du postulat, hérité de la sémiologie structuraliste des années 60-70 et inlassablement réitéré depuis, à savoir que le sens est immanent au matériau linguistique. L'appel à unenouvelle lecture, qui correspond à l'idée peir rcéenne dela sémiosejamais achevée dessignes, reposesur leprincipe du nécessai re renouvellement des savoirs déjà constitués. Peirce employait le mot «habitude» précisément pour désigner ces acquis de savoir et de culture; il faudrait cependant rappeler ce fait significatif que, lorsque Peirce emploie le mot «habitude», il l'utilise presque toujours dansl'expression «changement d'habitude» («habit change»). Les acquis marqués par ce dépassement du seuil linguistiquedans une situation sémiotique donnée, seront par la suite récupérés par les systèmes codifiés de référence, tels que la langue: c'est ainsi que les mouvements de la semiosis marquent toujours un moment ou une instance de désé quilibre permettant l'enrichissement de la langue.
Pour moi, la question n'est pas de savoir si le poème, comme vous |'écrivez, «vise à faire évoluer » un niveau de conscience ou un état de savoir. N on plus que de prêter une «intention» semblableau poète. U ne desidées centrales quej'ai retenues de Peirce, c'est que l'avancée des niveaux de conscience et des états de savoir, d'une part, et les processus de la semiosis, d'autre part, marqués dans le cas qui nous occupe ici par des lectures renouvelées qui nécessairement débordent le seuil linguistique, sont un seule et même processus. Pour l'exprimer en termes hjelmsléviens, je suggérerais qu'on trouve là la fonction sémi otique essentielle: avancée sémiosique des signes et gains de savoir et de conscience. D 'une certaine façon, le texte lu fournit des images, des indices et autres matériaux qui contribueront au surgissement d'un nouveau signe. Au moment où Roland Barthes débordait le projet sémiologique, au début des années 70 , il écrivait - je cite de mémoire- que le signe est un départ. D 'une certaine façon, cette idée de «départ» ouvre la voie aux divers mouvements de la semiosis.

Je reviens maintenant de façon plus précise à votre proposition concernant la lecture du poèmeet l'enchaînement des signes. À partir du moment où I'on reconnaît que la signification est un processus qui, débordant le seuil linguistique, trouve donc sa réalisation au-delà de la stricte matérialité manifestée de signes, il me semblerait inconséquent de rechercher, à l'intérieur du poème, des repères répartis, comme vous le suggérez au début, au milieu et à la fin du texteet qui correspondrai ent à une mise en scène ou à une représentation de ce processus de sémiose.

L'exemple que vous suggérez des coups frappés à la porte me paraît plus proche de la problématique de la sémiose, car il présentel'avantage de poser les questions sémiotiques de base d'unefaçon particulièrement claire. J evoudrais d'abord souligner lefait que derrièrela situation d'une occurrence simple d'un signe, vous introduisez, au-delà de la question de la reconnaissance d'un signe, celle de la codification établie sur la base d'une reconnaissance et d'un consensus qui s'établit entre le locuteur et l'interlocuteur. En somme vous mettez en scènele processus dela conventionnalisation; et vous avez parfaitement raison car c'est à ceniveau que se situe la problé matique essentielle de la sémiotique. C'est certainement une habitudetrès néfaste, que nous avons héritée de la sémiologie des codes, que celle de prendre pour acquis que les signes sont déjà et toujours exhaustivement codifiés comme de pleines positivités alors quel'on fait à chaque jour l'expérience de la nécessaire carence des signes, de leur négativité, de leur 
ouverture, bref de tous ces traits qui permettent aux signes une prise sur le réel.

Avec cet exemple, vous suggérez que la fonction sémiotique, faisant des coups frappés à la porte un signifiant renvoyant au signifié «demande d'ouverture», ne s'établit que sur la base d'un échange entreles deux interlocuteurs, d'une reconnaissance mutuelle et d'une connivence ou d'un accord sur la convention qui fonde cettefonction sémiotique. J e partage cetteanalyse sans réserve d'autant plus qu'elle rejoint le thème de la socialité de la signification auquel on s'est précédemment référé.

Je ne voudrais cependant pas entrer dans la mécanique des équivalences entre les strates du tableau hjelmslévien du signe et les moments successifs du processus de la semiosis. Sur ce plan précis, il est évident que vous êtes plus compétent que moi pour juger de la justesse de ces équivalences, en ce qui concerne le volet hjelmslévien. Je proposerai tout de même deux réflexions. La sémiotique hjelmslévienne a toujours été analysée comme une sémiologie des systèmes, c'est-à-dire une formalisation des codes de signes exhaustivement réalisés, ce qui correspond d'ailleurs parfaitement à la filiation saussurienne dont se réclamait $\mathrm{H}$ jelmslev lui-même. En ce sens, votre lecture est certainement, comme vous l'écrivez, «non orthodoxe»; par contre, j'imaginefacilement que ce soit là la seule façon dont on puisse aujourd'hui encore lire $\mathrm{H}$ jelmslev. Cependant cette relecture pose une difficulté majeure: c'est que les dénominations techniques comme substance du contenu ou substancedel'expression connaissent nécessairement des déplacements qui risquent de rendre leur usage problématique, sinon contradictoire. D eplus, vous devez introduire de nouvelles fonctions (dans votre exemple, l'action d'ouvrir la porte est un interprétant des coups frappés) qui, je pense, trouvent difficilement leur place sur les strates du tableau hjelmslévien du signe.

Par contre, et c'est ma deuxième réflexion, je crois que votre lecture de $\mathrm{H}$ jelmslev est assez proche de celle que suggérait Eco lorsque, dans Sémiotiqueet philosophiedu langage, il tentait d'établir une continuitéentre les deux substances du contenu et de l'expression; ce qui le conduisait à cette proposition assez étonnantequejereformule de mémoire: la matière dont est fait le signe et la matière dont il parle sont une seule et même chose. Il est assez significatif qu'Eco n'ait pas hésitéà affirmer que cette lecture était marquée de l'influence de sa découverte, encore récente à l'époque, de Peirce. Je reviens au poème, à la lecture que j'en ai proposée et à votreinterprétation de cettelecture. V ous écrivez: «[...] n'ai- je pas un peu raison de dire que, de mon point de vue, votre lecture de la finale correspond dans mon langage à moi à la forme du contenu ou au signifié en tant que ce qui manquait au signifiant pour qu'il puissey avoir signification sous forme de «serait», c'est-à-dire à la condition que quel qu'un d'autre en effectue la lecture?»

Je répondrai «0 ui et non!» et je regrette cette réponse de N ormand, maisjene puisfairemieux: prenant commeacquis que la lecture que l'on fait d'un poème (ou de tout autre texte) est un processus de sémiose qui réalise le «serait» du signe, c'est-à-dire qui le met en marche, lui apportant un prolongement, donc une avancée et un gain de signification, jerépondrai «non » dansla mesure où, suivant les définitions strictes qu'en donnent Saussure et $\mathrm{H}$ jelmslev, le «signifié» et la «formedu contenu » sont deséléments qui sont codifiés, intégrés dans un système défini contre toute forme de diachronieet un systèmene laissant aucune marge d'indécision au sujet pour son imaginaireet sa créativité; maisjerépondrai «oui » dans la mesure où la lecture, c'est une procédure qui apporteun nouveau signifiéau texte de départ et que, sansce nouveau signifié, le signe-texte resterait en attente d'une réalisation sémiosique. Ici, la différence entre Saussure H jelmslev d'une part et Peirce de l'autre, c'est que dans la semeiotic, ce que vous appelez le signifié, c'est un signe ultérieur en voie de surgissement qui dépassera celui qui le précèdeet le génèreet non pas un effet de retour sur lesigne, au sens du terme «boucle» que vous employez à plusieurs reprises et qui me paraît venir contredire cetteidée d'avancée et de débordement.

Je pense que la question centrale que vous posez revient à demander si le «signifié» ou la «forme du contenu» pourraient être assimilés, dans la problématique peircéenne, à I'«interprétant». J'ai répondu «oui » et «non ». Je répondrais positivement dans la mesure où les phénomènes de signification auxquels s'intéressent tous les sémioticiens, de quelque école et de quelque époque qu'ils soient, sont les mêmes et que les solutions proposées ne peuvent pas être totalement étrangères les unes aux autres. $M$ ais je vous répondrais par la négative dans la mesure où nous devons observer la plus grande rigueur dans la reconnaissance des diverses problématiques et dans l'usage des diverses terminologies qui leur sont liées. C'est làunequestion d'éthique, car un relâchement de cette exigence formelle risquerait de conduire les diverses théories sémiotiques dans un chaos notionnel où se perdrait ce qui fait leur spécificité et leur finesse respectives. 